\section{Are the antimicrobial properties of macrolides required for their therapeutic efficacy in chronic neutrophilic airway diseases?}

\author{
Guy G Brusselle 1,2
}

Macrolide antibiotics have been demonstrated to be efficacious in diverse chronic airway diseases characterised by neutrophilic airway inflammation, encompassing cystic fibrosis (CF), non-CF bronchiectasis, diffuse panbronchiolitis, exacerbationprone COPD and neutrophilic severe asthma. ${ }^{1-8}$ Many exogenous and endogenous factors can elicit airway neutrophilia: infections (bacterial, viral, virus-like ...), pollution (smoking, indoor and outdoor air pollution) and other factors (eg, autoimmunity, gastro-oesophageal reflux with aspiration). Macrolides such as erythromycin, clarithromycin and azithromycin not only have antimicrobial properties, but also broad anti-inflammatory and immunomodulatory effects. ${ }^{9}$ The antimicrobial effects of macrolides include direct bacterial killing, the prevention of biofilm formation by interfering with microbial quorum sensing, and the stimulation of phagocytosis of bacteria by macrophages. However, chronic use of macrolides has been associated with the occurrence of macrolide-resistant bacteria in the commensal flora of the pharynx of individual patients, and also induces the risk of an increase in antibiotic resistance at the population level. ${ }^{10}$ Therefore, it is crucial to address the research question whether the antimicrobial properties of macrolides are necessary for their therapeutic efficacy in chronic neutrophilic airway diseases. If not, then the development and use of nonantibiotic macrolides is warranted, since these novel drugs would retain the therapeutic efficacy without the risk of inducing microbial resistance.

In the current issue of Thorax, two complimentary papers address thoroughly the therapeutic potential of macrolides in two different chronic airway diseases which represent a high unmet medical need:

'Department of Respiratory Medicine, Ghent University Hospital, Ghent, Belgium; ${ }^{2}$ Department of Epidemiology and Respiratory Medicine, Erasmus Medical Center, Rotterdam, The Netherlands

Correspondence to Guy G Brusselle, Department of Respiratory Medicine, Ghent University Hospital, Ghent 9000, Belgium; guy.brusselle@ugent.be bronchiolitis obliterans syndrome (BOS) after lung transplantation and steroid-resistant severe asthma. In the first paper, Corris et $a l^{11}$ performed the first randomised controlled trial of azithromycin in the treatment of BOS post lung transplantation. In the second paper, Essilfie et $a l^{12}$ investigated the effects of clarithromycin in two mouse models of steroid-resistant asthma, and investigated in depth the mechanisms of action.

BOS is a major complication of lung transplantation and is associated with poor survival. According to the 2014 International Society for Heart and Lung Transplantation (ISHLT)/American Thoracic Society (ATS)/European Respiratory Society (ERS) international clinical practice guideline on the diagnosis and management of BOS, BOS is defined as a delayed allograft dysfunction with persistent decline in $\mathrm{FEV}_{1}$ that is not caused by other known and potentially reversible causes of loss of lung function after lung transplantation. ${ }^{13}$ Observational studies of treatment of patients with BOS and macrolides, in particular low-dose azithromycin, have suggested that one-third of patients may respond clinically with a gain of lung function and even a better life expectancy. ${ }^{14-16}$ However, since randomised controlled clinical trials (RCTs) of macrolides in the treatment of BOS have been lacking, the 2014 ISHLT/ATS/ERS guideline only 'suggests'instead of 'recommends' - a trial of azithromycin for lung transplant recipients who develop BOS (conditional recommendation, very low quality of evidence). ${ }^{13}$

In this issue of Thorax, Corris et al ${ }^{11}$ report the results of the first RCT of azithromycin therapy in BOS after lung transplantation. Forty-eight patients with BOS were randomised: 25 patients to azithromycin (250 mg alternate days, 12 weeks) and 23 to placebo. The primary outcome measure was the change in $\mathrm{FEV}_{1}$ from baseline to 12 weeks, whereas the change in bronchoalveolar lavage (BAL) neutrophils was a secondary outcome measure. In the intention to treat (ITT) analysis, the estimated mean difference in $\mathrm{FEV}_{1}$ between treatment groups (azithromycin minus placebo) was $0.035 \mathrm{~L}$ and nonsignificant $(95 \%$ CI -0.112 to $0.182 \mathrm{~L}$; $\mathrm{p}=0.6$ ). However, in the per protocol analysis of study completers, the mean difference in $\mathrm{FEV}_{1}$ between treatments was $0.278 \mathrm{~L} \quad(95 \%$ CI 0.170 to $0.386 \mathrm{~L}$; $\mathrm{p}<0.001)$. Moreover, patients who had a rapid and severe deterioration in lung function were withdrawn from the study, and were subsequently treated according to the usual centre and international practice, including the use of open-label azithromycin. Five withdrawals, who had been randomised to placebo, had received rescue open-label azithromycin, with subsequent improvement in $\mathrm{FEV}_{1}$ at 12 weeks. In a post hoc 'as treated' analysis (where the 5 withdrawal patients contributed to the overall azithromycin treatment), the mean difference in $\mathrm{FEV}_{1}$ between treatment groups (azithromycin minus placebo) was $0.306 \mathrm{~L}(\mathrm{p}<0.001)$. Nine of 23 ITT patients in the azithromycin group had a $\geq 10 \%$ gain in $\mathrm{FEV}_{1}$ from baseline, whereas no patients in the placebo group had $\mathrm{a} \geq 10 \%$ gain in $\mathrm{FEV}_{1}$ from baseline while on placebo. Finally, treatment of BOS with azithromycin was not associated with significant adverse events. ${ }^{11}$

This RCT strengthens the evidence that azithromycin therapy improves lung function in patients with BOS, and nicely compliments the RCT of azithromycin prophylaxis in lung transplant patients to prevent BOS. ${ }^{17}$ In the latter RCT, azithromycin significantly prolonged BOS-free survival; and patients receiving azithromycin had better $\mathrm{FEV}_{1}$ and lower airway neutrophilia. In the current RCT by Corris et al, however, azithromycin treatment was not associated with systematic changes in the percentage of BAL neutrophils. This contrasts with the findings in the observational study by Verleden et al, ${ }^{18}$ demonstrating that azithromycin treatment reduced airway neutrophilia and interleukin-8 (IL-8) in patients with BOS. It would be interesting to know whether the presence of BAL neutrophilia in the patients with BOS at baseline predicted the therapeutic response to azithromycin in the RCT by Corris. A predictor of therapeutic response is indeed not synonymous of a therapeutic target, as is illustrated with exhaled nitric oxide (FeNO) in asthma: an increased $\mathrm{FeNO}$ - as a marker of eosinophilic airway inflammation-is a predictor of response to treatment with inhaled corticosteroids (ICS); however, targeting inducible nitric oxide synthase-the enzyme responsible for the production of nitric oxide in airway epithelial cells-did not benefit patients with allergic asthma, as examined in the classical allergen 
challenge model. Similarly, the presence of airway or BAL neutrophilia in lung transplant patients might predict therapeutic response to macrolides, ${ }^{19}$ whereas the therapeutic target could be an underlying viral or bacterial infection, or gastrooesophageal reflux with microaspiration. Since macrolides promote gastric motility, prevention of reflux and aspiration might be an additional mechanism of action.

Severe asthma is a heterogeneous disease and encompasses several inflammatory phenotypes, including eosinophilic asthma, neutrophilic asthma and paucigranulocytic asthma. ${ }^{20} 21$ The time of onset of asthma (early onset in childhood vs late onset in adulthood) and the presence or absence of allergy are additional important determinants of the diverse clinical phenotypes of asthma. In classical mouse models of 'asthma', the investigators sensitise T helper 2 (Th2)-prone Balb/ c mice to experimental allergens such as ovalbumin (OVA) by intraperitoneal injection, and-2-6 weeks later-expose the sensitised animals to OVA via the inhaled route (ie, intranasal instillation or aerosol challenge). This leads to a Th2 adaptive immune response (characterised by the cytokines IL-4, IL-5 and IL-13), an eosinophilic airway inflammation and airway hyperresponsiveness (AHR) to methacholine. This classical mouse model of experimental asthma thus mainly mimics early-onset allergic eosinophilic asthma, which is driven by Th2 cells and is sensitive to corticosteroids (ICS in patients with asthma; dexamethasone in mice).

In their elegant study of macrolide therapy in experimental asthma, Hansbro and colleagues developed a novel model of steroid-resistant asthma, by infecting OVA-sensitised mice with Chlamydia via intranasal instillation. ${ }^{12}$ Importantly, this combination of allergen (OVA) exposure and infection (Chlamydia) increased airway neutrophils, tumour necrosis factor (TNF- $\alpha$ ) responses and AHR, which were all insensitive to treatment with corticosteroids. To disentangle the antibiotic effects from the anti-inflammatory effects of macrolides, the investigators compared the in vivo effects of clarithromycin with those of amoxicillin. In the combined OVA-Chlamydia model, treatment with amoxicillin did not affect total BAL cells and AHR, whereas clarithromycin significantly reduced total cells, neutrophils and macrophages in BAL fluid, and suppressed both TNF- $\alpha$ release and AHR. Moreover, the authors confirmed that treatment with clarithromycin decreased BAL neutrophils and AHR in a second model of steroid-resistant asthma, induced by intratracheal infection with Haemophilus influenzae $(\mathrm{Hi})$ before sensitisation to OVA. Intriguingly, in the $H i$-induced steroid-resistant asthma model, clarithromycin reduced IL-17 levels in mediastinal lymph node cultures. IL-17, a cytokine produced by $\mathrm{T}$ helper 17 cells and several innate immune cells, induces neutrophilic airway inflammation by inducing IL-8 production by epithelial cells. IL-17 has two faces: it plays an important protective role in host defence against extracellular bacteria (such as $\mathrm{Hi}$ ), but has also been linked to auto-immunity.

These preclinical studies by Hansbro and colleagues indicate that clarithromycin has antimicrobial and mainly broad antiinflammatory effects, reducing key mediators (eg, TNF- $\alpha$ and IL-17) in models of steroid-resistant neurophilic asthma. Can we translate these murine observations to human patients with (severe) asthma? Yes, since clarithromycin has also been shown to reduce airway neutrophil numbers, sputum IL-8 and neutrophil elastase in patients with steroid-resistant asthma. ${ }^{22}$ Probably not (entirely), if we take into account the numerous differences between the murine models and humans with asthma. First, the timing of the intervention with macrolides is different: in the murine model, clarithromycin is administered before the sensitisation to OVA (in the Hi-OVA model) or 2 weeks before the final intranasal OVA challenges (in the OVA-Chlamydia model), implicating that this approach is a preventive strategy. In contrast, patients with asthma seek medical attention with chronic complaints of cough and shortness of breath, long after the underlying airway inflammation has been fully established. In this clinical scenario, physicians can only offer therapeutic strategies (as opposed to prevention in animal models). Second, although OVA is the most widespread used allergen in murine models of asthma, it is an irrelevant innocuous aero-antigen for patients with asthma. In people with allergic asthma, house dust mite is indeed the most prevalent causative allergen. Lastly, in addition to the evident differences in anatomy, physiology, immunology, microbiome and genetics between mice and humans, the endpoints which are investigated are fundamentally different: immunologic endpoints (eg, inflammatory cells in BAL) in murine models versus symptoms, patient-reported outcomes, exacerbations and lung function measurements (ie, clinical and physiologic endpoints) in human studies. In the AZISAST RCT, maintenance treatment of patients with non-eosinophilic severe asthma with azithromycin significantly reduced the rate of exacerbations, the primary outcome of this trial. ${ }^{5}$

In summary, by performing a RCT of azithromycin treatment of BOS after lung transplantation, Corris et $a l^{11}$ have added lung allograft BOS to the expanding list of indications for chronic low-dose macrolide therapy in respiratory medicine. In the domain of experimental asthma, Essilfie et $a l^{12}$ have developed valuable mouse models of steroid-resistant neutrophilic asthma by combining allergen exposures with bacterial infections. In these combined models, the immunomodulatory properties of macrolides appear to be the predominant mechanism of action. In patients with severe neutrophilic asthma, COPD, CF, non-CF bronchiectasis and BOS, I put forward the hypothesis that the pathogenic mechanisms which drive the neutrophilic airway inflammation will determine whether the patient will respond to macrolide treatment and, if positive, whether the antimicrobial or antiinflammatory properties of the macrolide are critical for its therapeutic efficacy.

\section{Competing interests None.}

Provenance and peer review Commissioned; internally peer reviewed.

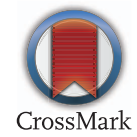

To cite Brusselle GG. Thorax 2015;70:401-402. Accepted 20 March 2015

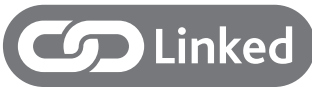

http://dx.doi.org/10.1136/thoraxinl-2014-205998 http://dx.doi.org/10.1136/thoraxjn-2014-206067

Thorax 2015;70:401-402.

doi:10.1136/thoraxjnl-2015-207080

\section{REFERENCES}

1 Equi A, Balfour-Lynn IM, Bush A, et al. Long term azithromycin in children with cystic fibrosis: a randomised, placebo-controlled crossover trial. Lancet 2002;360:978-84.

2 Wong C, Jayaram L, Karalus $\mathrm{N}$, et al. Azithromycin for prevention of exacerbations in non-cystic fibrosis bronchiectasis (EMBRACE): a randomised, double-blind, placebo-controlled trial. Lancet 2012;380:660-7.

3 Albert RK, Connett J, Bailey WC, et al. Azithromycin for prevention of exacerbations of COPD. $N$ Engl J Med 2011;365:689-98.

4 Altenburg J, de Graaff CS, Stienstra Y, et al. Effect of azithromycin maintenance treatment on infectious exacerbations among patients with non-cystic fibrosis bronchiectasis: the bat randomized controlled trial. JAMA 2013;309:1251-9.

5 Brusselle GG, VanderStichele C, Jordens $P$, et al. Azithromycin for prevention of exacerbations in severe asthma (AZISAST): a multicentre randomised 
double-blind placebo-controlled trial. Thorax 2013;68:322-9.

6 Koyama H, Geddes DM. Erythromycin and diffuse panbronchiolitis. Thorax 1997;52:915-18.

7 Crosbie PAJ, Woodhead MA. Long-term macrolide therapy in chronic inflammatory airway diseases. Eur Respir J 2009;33:171-81.

8 Brusselle GG, Joos $\mathrm{G}$. Is there a role for macrolides in severe asthma? Curr Opin Pulm Med 2014;20:95-102.

9 Lopez-Boado YS, Rubin BK. Macrolides as immunomodulatory medications for the therapy of chronic lung diseases. Curr Opin Pharmacol 2008;8:286-91.

10 Serisier D. Risks of population antimicrobial resistance associated with chronic macrolide use for inflammatory airway diseases. Lancet Respir Med 2013;1:262-74.

11 Corris P, Ryan VA, Small T, et al. A randomised controlled trial of azithromycin therapy in Bronchiolitis Obliterans Syndrome (BOS) post lung transplantation. Thorax 2015;70:442-50.
12 Essilfie A-T, Horvat J, Kim R, et al. Macrolide therapy suppresses key features of experimental steroid-sensitive and steroid-insensitive asthma. Thorax 2015;70:458-67.

13 Meyer KC, Raghu G, Verleden GM, et al. An international ISHLT/ATS/ERS clinical practice guideline: diagnosis and management of bronchiolitis obliterans syndrome. Eur Respir I 2014;44:1479-503.

14 Gerhardt SG, McDyer JF, Girgis RE, et al. Maintenance azithromycin therapy for bronchiolitis obliterans syndrome. Am J Respir Crit Care Med 2003; 168:121-5.

15 Yates B, Murphy DM, Forrest IA, et al. Azithromycin reverses airflow obstruction in established bronchiolitis obliterans syndrome. Am I Respir Crit Care Med 2005;172:772-5.

16 Jain R, Hachem RR, Morrell MR, et al. Azithromycin is associated with increased survival in lung transplant recipients with bronchiolitis obliterans syndrome. J Heart Lung Transplant 2010;29:531-7.

17 Vos R, Vanaudenaerde BM, Verleden SE, et al. A randomised controlled trial of azithromycin to prevent chronic rejection after lung transplantation. Eur Respir J 2011;37:164-72.

18 Verleden GM, Vanaudenaerde BM, Dupont LJ, et al. Azithromycin reduces airway neutrophilia and interleukin-8 in patients with bronchiolitis obliterans syndrome. Am J Respir Crit Care Med 2006;174:566-70.

19 Vanaudenaerde BM, Meyts I, Vos R, et al. A dichotomy in bronchiolitis obliterans syndrome after lung transplantation revealed by azithromycin therapy. Eur Respir J 2008;32:832-43.

20 Wenzel SE. Asthma phenotypes: the evolution from clinical to molecular approaches. Nat Med 2012;18:716-25.

21 Chung KF, Wenzel SE, Brozek JL, et al. International ERS/ATS guidelines on definition, evaluation and treatment of severe asthma. Eur Respir J 2014;43: 343-73.

22 Simpson J, Powell H, Boyle M, et al. Clarithromycin targets neutrophilic airway inflammation in refractory asthma. Am I Respir Crit Care Med 2008;177:148-55. 